\title{
Hepatotoxic Potential of Therapeutic Oligonucleotides Can Be Predicted from Their Sequence and Modification Pattern
}

\author{
Peter H. Hagedorn, ${ }^{1}$ Victor Yakimov, ${ }^{2}$ Søren Ottosen, ${ }^{3}$ Susanne Kammler, ${ }^{3}$ Niels F. Nielsen, ${ }^{3}$ \\ Anja M. Høg, ${ }^{3}$ Maj Hedtjärn, ${ }^{3}$ Michael Meldgaard, ${ }^{4}$ Marianne R. Møller, ${ }^{5}$ Henrik Ørum, ${ }^{6}$ \\ Troels Koch, ${ }^{6}$ and Morten Lindow ${ }^{1,2}$
}

Antisense oligonucleotides that recruit RNase $\mathrm{H}$ and thereby cleave complementary messenger RNAs are being developed as therapeutics. Dose-dependent hepatic changes associated with hepatocyte necrosis and increases in serum alanine-aminotransferase levels have been observed after treatment with certain oligonucleotides. Although general mechanisms for drug-induced hepatic injury are known, the characteristics of oligonucleotides that determine their hepatotoxic potential are not well understood. Here, we present a comprehensive analysis of the hepatotoxic potential of locked nucleic acid-modified oligonucleotides in mice. We developed a random forests classifier, in which oligonucleotides are regarded as being composed of dinucleotide units, which distinguished between 206 oligonucleotides with high and low hepatotoxic potential with 80\% accuracy as estimated by out-of-bag validation. In a validation set, 17 out of 23 oligonucleotides were correctly predicted $(74 \%$ accuracy). In isolation, some dinucleotide units increase, and others decrease, the hepatotoxic potential of the oligonucleotides within which they are found. However, a complex interplay between all parts of an oligonucleotide can influence the hepatotoxic potential. Using the classifier, we demonstrate how an oligonucleotide with otherwise high hepatotoxic potential can be efficiently redesigned to abate hepatotoxic potential. These insights establish analysis of sequence and modification patterns as a powerful tool in the preclinical discovery process for oligonucleotide-based medicines.

\section{Introduction}

A N ESSENTIAL PROPERTY when developing oligonucleotides for therapeutics is that their main interactions with RNA follows Watson and Crick's base pairing rules for nucleic acids (Bennet and Swayze, 2010). Given these rules, and the sequence of an RNA molecule, designing perfectly matching oligonucleotides is straightforward. When using modern nucleic acid modification chemistries such as highaffinity locked nucleic acids (LNAs) (Obika et al., 1997; Koshkin et al., 1998) or 2'-O-methoxy ethyl (2'MOE) (Bennet and Swayze, 2010), in combination with a phosphorothioate backbone (Stein et al., 1998), a large fraction of such designs are able to bind and inhibit the targeted RNA. In contrast, for small molecules targeting proteins, screening libraries often need to contain hundreds of thousands of compounds in order to identify hits against a protein target (Hert et al., 2009).
This makes oligonucleotides targeting RNA very attractive when it comes to fast and cost-effective discovery of efficacious and potent drug candidates.

Currently, the requirements for regulatory approval of oligonucleotides and small molecule drugs are similar (Schubert et al., 2012). Therefore, when it comes to effects not related to Watson-Crick guided hybridization, such as toxic liabilities, oligonucleotides and small molecule drugs are screened in a similar manner. As yet, very few oligonucleotides are on the market, but clinical and preclinical adverse effects reported for high-affinity oligonucleotides developed in recent years include injury to the liver and kidneys, two primary organs of oligonucleotide accumulation, as well as injection site reactions (Levin, 1999; Henry et al., 2007; Bennet and Swayze, 2010; Lindow et al., 2012). It seems that oligonucleotides as a chemical class are particularly associated with these types of toxicities. However, for any single

Departments of ${ }^{1}$ Informatics, ${ }^{3}$ Technology and Partnered Discovery, ${ }^{4}$ Chemistry and CMC, and ${ }^{5}$ In vivo Pharmacology, ${ }^{6}$ Santaris Pharma A/S, Hørsholm, Denmark.

${ }^{2}$ The Bioinformatics Centre, Department of Biology, University of Copenhagen, Copenhagen, Denmark. 
oligonucleotide, irrespective of its modification-chemistry, the degree to which it manifests any of these liabilities, if at all, varies widely. In the case of hepatotoxicity, specific oligonucleotides with LNA modifications (Swayze et al., 2007; Seth et al., 2009; Stanton et al., 2012) and 2'MOE modifications (Swayze and Siwkowski, 2009; Burel and Henry, 2010) have been reported to induce elevations in alanine-aminotransferase (ALT), a serum biomarker of hepatocellular injury, when administered to mice even at relatively low doses. But on the other hand, many well-tolerated LNA-modified as well as 2'MOE-modified oligonucleotides have also been reported where no dose-limiting hepatotoxicity were seen during preclinical and clinical testing (Elmén et al., 2008; Bennet and Swayze, 2010; Gupta et al., 2010; Straarup et al., 2010; Janssen et al., 2011; Hildebrandt-Eriksen et al., 2012; Lindholm et al., 2012). These examples illustrate the marked differences in the hepatotoxic potential of different oligonucleotide compounds.

Discovering the characteristics of compounds that are more likely to yield safe, potent, and efficacious drugs is central for the development of drug discovery into a knowledge-based predictive science (Lipinski and Hopkins, 2004). Attempts to quantify such structure-activity relationships for small molecule compounds have shown good predictivity for specific endpoints such as solubility and permeability (Lipinski et al., 2001) or binding affinity to proteins with a known 3-dimensional structure (Wang et al., 2002). For complex endpoints such as hepatotoxicity, however, the predictivity remains poor (Low et al., 2011). Deriving descriptors from the chemical structure of small molecules that can be related to their toxic potential is not trivial (Benigni and Giuliana, 2003).

In this work, we set out to investigate whether structural descriptors of LNA-modified oligonucleotides can explain a complex endpoint, such as their hepatotoxic potential. Decomposing the chemical structure of an oligonucleotide into its nucleobase sequence and modification pattern, we report that machine learning techniques can produce a classification scheme, that captures a large part of the variation in the hepatotoxic potential of these oligonucleotides.

\section{Materials and Methods}

\section{Oligonucleotides}

LNA-modified antisense oligonucleotides were synthesized with complete phosphorothioate backbones using standard phosphoramidite protocols on an ÄKTA Oligopilot (GE Healthcare). After synthesis, the oligonucleotides were deprotected and cleaved from the solid support using aqueous ammonia at $65^{\circ} \mathrm{C}$ overnight. The oligonucleotides were purified by ion exchange high-performance liquid chromatography by applying a gradient of buffer B: $0 \%-80 \%$ in 38 column volumes (CV) followed by a washout of the column with $100 \%$ buffer B (4CV) and $100 \%$ buffer C (4 CV) before reequilibration with $100 \%$ buffer $\mathrm{A}(4 \mathrm{CV})$. The applied buffers were A: $10 \mathrm{mM} \mathrm{NaOH}$; B: $10 \mathrm{mM} \mathrm{NaOH} ; 2 \mathrm{M} \mathrm{NaCl}$; and C: $\mathrm{B} / \mathrm{EtOH} 4: 1$. The collected fractions from the purification were analyzed by ultra-performance liquid chromatography and fractions with a purity above $85 \%$ were pooled and the $\mathrm{pH}$ of the pool adjusted with $\mathrm{HCl}(1 \mathrm{M})$ to $\mathrm{pH}$ 7-8. Subsequently, the pooled fractions were desalted on an Äkta CrossFlow using a Millipore membrane with a 1-kDa cutoff (Pellicon 2 Mini Ultrafiltration Module PLAC C $0.1 \mathrm{~m}^{2}$ ) and lyophilized. Finally, liquid chromatography-mass spectrometry (reverse phase and electrospray ionization-mass spectrometry) was used to verify compound identity and purity.

\section{Animal experiments}

Inbred C57BL/6J and outbred NMRI female mice were obtained from Taconic (Denmark) and fed standard diet ad libitum. At study start, C57BL/6J mice weighed $20 \pm 2 \mathrm{~g}$ (arithmetic mean \pm standard deviation) and NMRI mice $24 \pm 2 \mathrm{~g}$. The animal facility was maintained on a 12 -hour light-dark cycle throughout each study. In 25 individual studies, mice were divided into treatment groups $(n=5)$ and dosed on days $0,3,7,10$, and 14 . The treatment groups received either $0.9 \%$ saline or saline-formulated antisense oligonucleotide administered by intravenous injection. On day 16 , mice were weighed, anesthetized $\left(70 \% \mathrm{CO}_{2} / 30 \% \mathrm{O}_{2}\right)$ before retroorbital blood sampling, and sacrificed by cervical dislocation. Livers were inspected visually and weighed. For real-time quantitative reverse transcription polymerase chain reaction (qRT-PCR), liver samples were immersed in RNAlater (Sigma-Aldrich). For histopathology, liver samples were fixed in formalin and embedded in paraffin (FFPE). All mouse protocols were approved by the Danish National Committee for Ethics in Animal Experiments.

\section{Serum alanine-aminotransferase analysis and defining the upper limit of normal}

Serum was analyzed for alanine-aminotransferase (ALT) activity using an enzymatic assay (Horiba ABX Diagnostics) according to the manufacturer's instructions adjusted to 96well format. Measurements were correlated to a 2-fold diluted standard curve generated from an ABX Pentra MultiCal solution (Horiba ABX Diagnostics). Within each study, the mean ALT activity in the saline treated group was calculated, and all ALT levels presented as fold changes relative to that mean value. From the distribution of ALT levels in all salinetreated mice across all studies, robust measures of central tendency and variability were calculated as median, $m=1.0$, and median absolute deviation, $\sigma=0.24$. From this, the ALT upper limit of normal was defined as ULN $=m+3 \sigma=1.7$.

\section{$R N A$ isolation and real-time quantitative RT-PCR}

Total RNA from liver was isolated using the RNeasy kit (Qiagen) and quantification of messenger RNA (mRNA) was done using TaqMan assays (Applied Biosystems). The reverse transcription reaction was carried out with random decamers, $0.5 \mathrm{mg}$ total RNA and the M-MLV reverse transcriptase enzyme (Ambion) according to protocol for first strand complementary DNA (cDNA) synthesis. Depending on expression levels, cDNA was subsequently diluted 5 times in nuclease-free water before addition to the RT-PCR reaction mixture. The Applied Biosystems 7500/7900/ViiA real-time PCR instruments were used for amplification. Within each study, mRNA levels were normalized to actin, beta (Actb) or glyceraldehyde-3phosphate dehydrogenase (Gapdh) and presented as foldchanges relative to average levels in saline controls.

\section{Histopathology}

Mouse FFPE liver samples were sectioned and stained with hematoxylin and eosin. Each section was examined by a 
single histopathologist and scored on 25 categories between 0 (absence of that change) and 3 (moderate to extensive) (Supplementary Table S1; Supplementary Data are available online at www.liebertpub.com/nat).

\section{Statistical modeling and sequence analysis}

The sequence composition and modification pattern of each oligonucleotide was encoded as an ordered feature vector of dinucleotide counts. With 4 DNA nucleotides (a, c, t, g) and 4 LNA variants $(A, C, T, G)$, the dimension of the feature vector is $(4+4)^{2}=64$. Each feature vector was assigned a class label as either high-tox or low-tox depending on the level of ALT measured when administering the corresponding oligonucleotide in mice (ALT $>5 \times$ ULN or ALT $<2 \times$ ULN, respectively). Feature vectors with associated class labels were used to train a random forests algorithm by growing unpruned classification trees from 5000 bootstrap samples of the training set (Breiman, 2001). For each tree, at each node, 8 out of the 64 dinucleotides were randomly selected, and the dinucleotide resulting in the best split chosen. The best split is defined as the split that maximally reduces the Gini impurity in the descendant nodes (Duda et al., 2001). For an ensemble of trees that have been trained in this manner, oligonucleotides to be classified are presented to each tree, and assigned the class voted for by the majority of trees (majority rule). An estimate of the accuracy of the prediction was calculated by averaging the prediction accuracies of each tree on the samples not included in training that tree (denoted out-of-bag samples). The accuracy of such an out-of-bag estimate has been demonstrated to be comparable to using a test set of the same size as the training set (Breiman, 2001). An estimate of the importance of each dinucleotide in the classification was calculated as the average decrease in Gini impurity over all splits where that dinucleotide was used (Breiman, 2001). Distance between sequences was calculated as the minimally possible number of substitutions, insertions, and deletions needed to transform one sequence into the other (generalized Levenshtein edit distance) (Levenshtein, 1966). Distance between a sequence and a group of sequences was calculated as the distance between the sequence and the closest member in the group of sequences (single linkage distance).

\section{Results}

\section{Characterizing and defining hepatotoxicity}

We systemically administered 236 different saline-formulated LNA-modified phosphorothioate antisense oligonucleotides by five intravenous injections of $15 \mathrm{mg} / \mathrm{kg}$ in C57BL/ $6 \mathrm{~J}$ or NMRI mice over a 16-day period. Following standard guidelines for hepatotoxicity screening in rodents (Peters, 2005), the total dose administered over approximately two weeks in this setup is more than 2-fold higher than the expected therapeutically relevant dose of a lead LNAmodified oligonucleotide, which is usually at $5-15 \mathrm{mg} / \mathrm{kg}$ a week in mice (Gupta et al., 2010; Lindholm et al., 2012; Straarup et al., 2010). About half of the oligonucleotides tested were found to induce liver injury to varying degrees as observed from highly elevated average levels of ALT in serum compared to saline-treated control mice 16 days after first dose (Fig. 1a). Histopathological evaluation of the livers from a subset of the mice confirmed that increases in
ALT were significantly associated with single-cell necrosis (Fig. 1b). In addition, periportal clumping of cytoplasm, scattered eosinophilic droplets, and formation of microgranuloma, were also significantly associated with elevated levels of ALT (Fig. 1b). Variation of the dose level for a subset of the oligonucleotides resulted in dose-dependent changes of the ALT levels as measured 16 days after first dose (Supplementary Fig. S1a). Similarly, variation of the sacrifice time revealed that measurements of ALT levels 3 days after first dose, keeping the total dose administered at $75 \mathrm{mg} / \mathrm{kg}$, were always lower than measurement of ALT levels 16 days after first dose (Supplementary Fig. S1b). Finally, the degree of hepatotoxicity observed generally correlated with increased liver/body weight ratio (Fig. 1c and Supplementary Fig. S1c, d), as have also been observed previously (Swayze et al., 2007; Henry et al., 2000). Taken together these observations qualify the utility of ALT as a biomarker for oligonucleotide-induced liver injury in mice. The 236 oligonucleotides in the data set were part of screening campaigns to find therapeutic candidates against 13 different mRNAs. For the targets where knock-down of the target mRNA was measured in the liver, no association between ALT levels and reduction in target mRNA levels was found (Fig. 1d). This suggests that the observed hepatotoxicity was not an effect of exaggerated on-target-pharmacology of the antisense oligonucleotides.

\section{Associating hepatotoxic potential with sequence}

To address whether sequence-based descriptors can be associated with the hepatotoxic potential, we grouped the 236 oligonucleotides based on the presence or absence of specific dinucleotides in their sequence, and examined the distribution of ALT levels in each group. Each oligonucleotide consists of a central region, the gap, of at least 7 DNA nucleotides, flanked by 2-3 LNA nucleotides at each end. Of the 16 possible dinucleotides appearing in the DNA-only gapregion of the oligonucleotides, 9 were found to significantly $(P<0.05)$ associate with either increased or decreased ALT levels (Fig. 2a). In contrast, of the 16 possible dinucleotides appearing in the LNA-only flank-regions of the oligonucleotides, only one, GT, was found to significantly associate with ALT (Fig. 2b).

\section{Predicting hepatotoxic potential from sequence}

The distribution of average ALT levels for each oligonucleotide in Fig. 1a displayed a peak around 1 (equal to average ALT level in intrastudy saline-treated control mice), representing oligonucleotides with low hepatotoxic potential, and a heavy tail at higher levels of ALT, representing progressively higher potential for hepatotoxicity. From this, oligonucleotides displaying less than two times ALT upper limit of normal (ULN) levels, clearly included all oligonucleotides with no or very low hepatotoxic potential (designated low-tox oligonucleotides), and oligonucleotides displaying more than five times elevated ULN levels were defined as having a clear high hepatotoxic potential (designated high-tox oligonucleotides), see Fig. 1a. We observed no difference in the prevalence of high-tox and low-tox oligonucleotides between NMRI and C57BL/6J mice (Supplementary Tables S2 and S3). Based on these observations, from the initial set of 236 oligonucleotides we generated reduced subsets of 97 and 109 


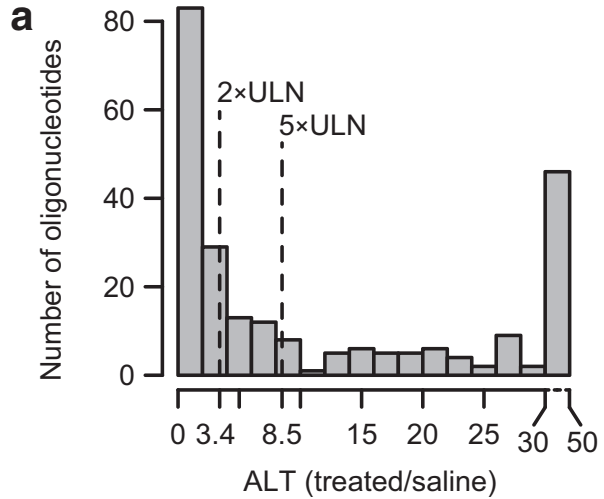

b

Single cell necrosis Periportal clumping of $(P<0.0001) \quad$ cytoplasm $(P<0.001)$

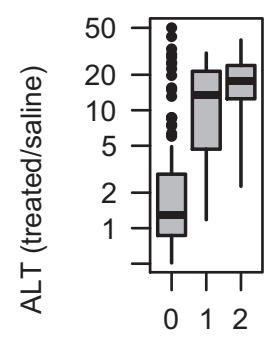

Scattered eosinophilic droplets $(P<0.001)$

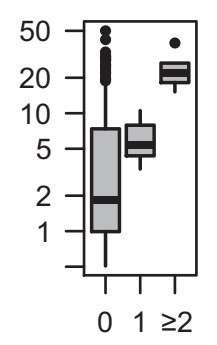

Microgranuloma $(P<0.05)$

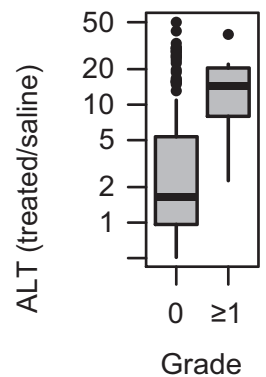

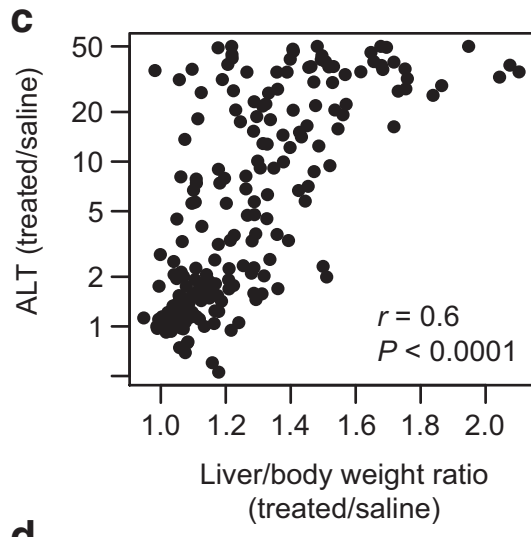

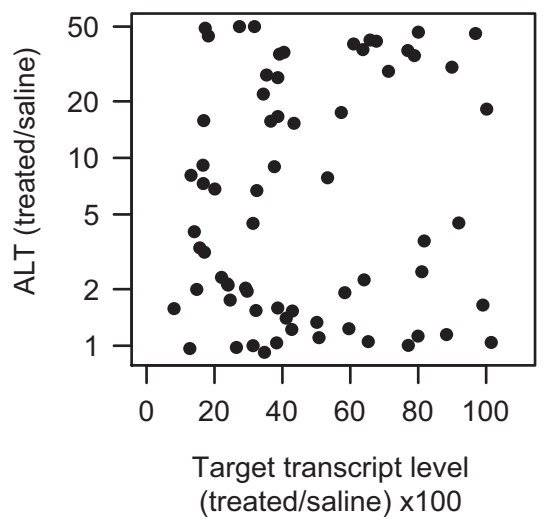

FIG. 1. Evaluation of the hepatotoxic potential of locked nucleic acid (LNA)-modified oligonucleotides in mice. (a) Distribution of average alanine-aminotransferase (ALT) levels $(n=5)$ relative to intrastudy average saline control mice $(n=5)$ for 236 LNA-modified oligonucleotides. Two- and five-times upper limit of normal (ULN) ALT levels are indicated. (b) Association between histopathological observables graded between 0 (nothing observed) and 3 (moderate to extensive) and ALT levels relative to saline in individual mice $[n=162$ mice treated with 29 oligonucleotides or saline; boxes cover interquartile range (IQR) and median, whiskers extend to $1.5 \times \mathrm{IQR} ; \quad P$ values calculated by one-way analysis of variance]. (c) Correlation between average liver/body weight ratio for mice treated with the same oligonucleotide and the average ALT level in these mice $(n=203$ oligonucleotides; Pearson's product-moment correlation). (d) For oligonucleotides targeting liver transcripts $(n=67$ oligonucleotides against 8 target transcripts), average ALT level in mice treated with the same oligonucleotide plotted against average liver target transcript level in these mice. oligonucleotides, respectively, by removing oligonucleotides with intermediate ALT levels (between $2 \times$ ULN and $5 \times$ ULN). For each sequence, an ordered feature vector of all 64 possible dinucleotide counts was computed and used to train a random forests classification algorithm (Breiman, 2001). Classification performance was estimated from out-of-bag samples at $80 \%$ accuracy $(76 \%$ specificity and $83 \%$ sensitivity; $P<0.001$ by Fisher's exact test; Fig. 3a). When evaluating the importance of each dinucleotide in the classification based on the average decrease in Gini impurity (Duda et al., 2001), 11 dinucleotides identified as significantly associated with hepatotoxicity when testing one feature at a time (as in Fig. 2a, b) were also identified as important in the random forests classification (Fig. 3b). Moreover, when several features are combined in the random forests, an additional set of at least 6 dinucleotides were revealed as being highly important (Fig. $3 b)$ for the classification accuracy. We systematically tested other encoding schemes, as well as Markov chains (Durbin et al., 1998) and support vector machines (Platt, 1999) as alternative classification algorithms, without seeing improved performance compared to the random forests classifier trained on dinucleotide counts presented here (Supplementary Table S4).

\section{Sequence distance from training set affects predictive performance}

To evaluate how the predictive performance of the random forests classifier is affected by the degree of similarity between the sequence to be predicted, and the sequences used to train the classifier, we first calculated Levenshtein edit-distances (Levenshtein, 1966) between all oligonucleotides in the training set. Next, we randomly divided the training set into 10 equally sized subsets, keeping the ratio of high-tox to lowtox oligonucleotides constant. The classifier was then trained 10 times, leaving a different subset out of the training in each round (the test set), and evaluating the performance of that round's trained classifier by predicting the class of the oligonucleotides in the test set. In addition, the distance between each of the oligonucleotides in the test set, and the closest oligonucleotide in that round's training set, was recorded. The predictive performance of the classifier as estimated in this 

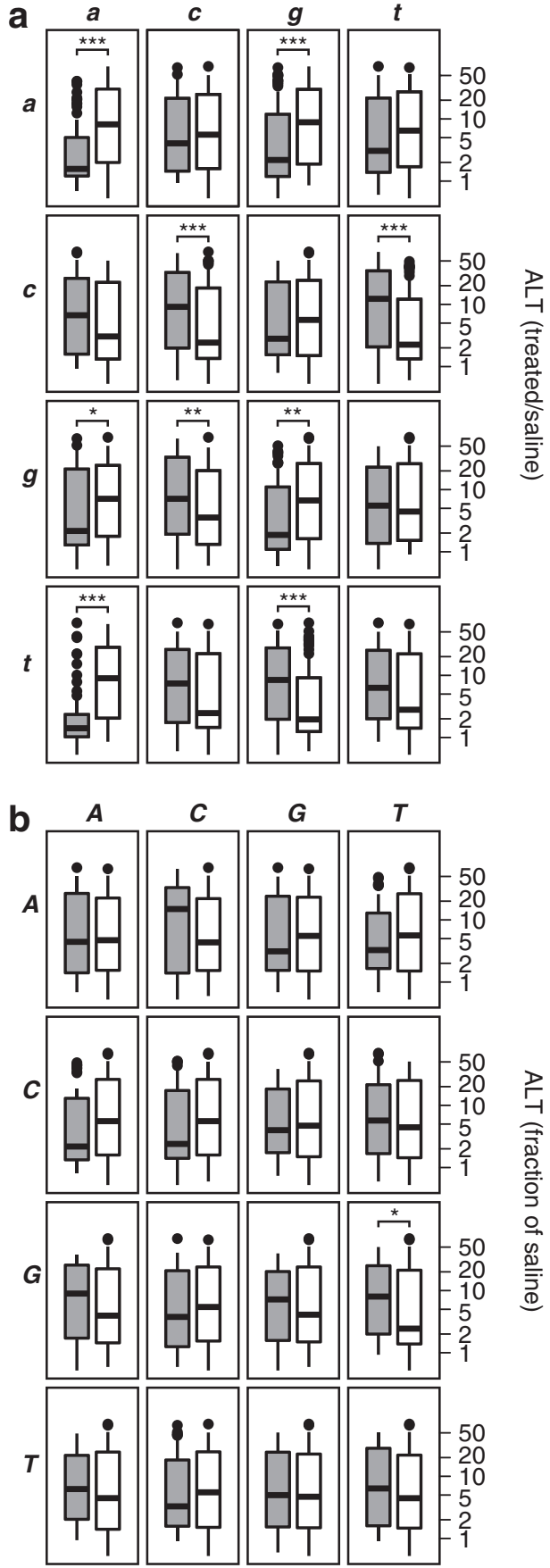

FIG. 2. Association between oligonucleotide sequence and hepatotoxic potential. (a) For each of the 16 possible DNA-only dinucleotides present in the gap-region of the oligonucleotides, the distributions of ALT levels for oligonucleotides where the dinucleotide is either present (gray) or absent (white) are shown $\left({ }^{*} P<0.05,{ }^{* *} P<0.01,{ }^{* * *} P<0.001\right.$, Wilcoxon rank-sum test). Note that $y$-axis is log-scale. (b) Same as Fig. 2a, but for LNA-only dinucleotides present in the flanks of the oligonucleotides.

manner (Fig. 3c) is highest for sequences that are very similar to one or more sequences in the training set (when only 1 or 2 substitutions, insertions, or deletions are needed), with an accuracy of $87 \%$ (83\% specificity and $90 \%$ sensitivity; $P<0.001$ by Fisher's exact test). For sequences that are further away (between 3 and 5 edits), the accuracy is estimated at $80 \%(82 \%$ specificity and $77 \%$ sensitivity; $P<0.001$ by Fisher's exact test). Finally, for sequences that are far away (between 6 and 8 edits), the accuracy is down to $69 \%$ (63\% specificity and $77 \%$ sensitivity; $P<0.01$ by Fisher's exact test). The slightly decreasing accuracy of the prediction as a function of distance suggests that the trained random forests algorithm does not generalize fully to all possible combinations of dinucleotide counts, and may improve further as more training data become available.

\section{Classification of oligonucleotides dosed at different levels}

In addition to the setup with $15 \mathrm{mg} / \mathrm{kg}$ dosed five times over 16 days used for screening (Fig. 1), we also tested other dose levels. Out of 51 oligonucleotides dosed five times over 16 days, but with a total dose below $75 \mathrm{mg} / \mathrm{kg}$ (between $25 \mathrm{mg} / \mathrm{kg}$ and $60 \mathrm{mg} / \mathrm{kg}$ ), 13 oligonucleotides displayed ALT increases above $5 \times$ ULN. Because of the observed doserelationship for hepatotoxicity (Supplementary Fig. S1a), these oligonucleotides would be expected to have at least as high ALT increases, had they been screened at $75 \mathrm{mg} / \mathrm{kg}$ total dose. Similarly, out of 33 oligonucleotides dosed five times over 16 days at higher than $75 \mathrm{mg} / \mathrm{kg}$ total dose (between $100 \mathrm{mg} / \mathrm{kg}$ and $125 \mathrm{mg} / \mathrm{kg}$ ), 10 oligonucleotides did not result in increases in ALT above $2 \times$ ULN. Using the same argument, had they been screened at $75 \mathrm{mg} / \mathrm{kg}$ total dose, the ALT levels would be expected to be just as low or even lower. None of these $13+10=23$ oligonucleotides were included in the set of oligonucleotides used to train the random forests classifier, and they target 10 different mRNAs, 8 of which are different from those used in the training set. Consequently, they can be used as a validation set to test the performance of the classifier. When the random forests classifier was applied to this independent set of oligonucleotides, an overall accuracy of $74 \%$ was achieved ( $69 \%$ specificity and $80 \%$ sensitivity; $P<0.05$ by Fisher's exact test; Fig. 3d). When calculating distances to the training set, most were between 5 and 7 edits away (Fig. 3e), and for these distances, a classification accuracy of $74 \%$ matches well the expected classification accuracy as estimated from 10-fold cross validation (Fig. 3c).

\section{Using the classifier in the efficient discovery of therapeutic oligonucleotides}

For oligonucleotides where the dose levels needed to achieve sufficient target knockdown lies close to the dose levels that result in hepatic injury, we asked whether the trained random forests classifier could be used to identify alternative designs with reduced hepatotoxic potential. To evaluate this, we choose a LNA-modified oligonucleotide, designated seth, which has been reported as hepatotoxic by Seth et al., 2009. Under the constraints that re-designed oligonucleotides should have at least two and at most four LNAs in each flank, be between $13 \mathrm{nt}$ and $16 \mathrm{nt}$ in length, and at most target a region 1nt up- or downstream of the original target region, 90 redesigns are possible (Supplementary Table S5). These redesign constraints were selected based on our experience with the types of designs that have resulted in potent and efficacious oligonucleotides. The distribution of hepatotoxicity prediction scores, when applying the random forests classifier to the redesigns is shown in Fig. 4a. The 
hepatotoxicity prediction score is the fraction of trees in the random forests that predicts an oligonucleotide as having a high hepatotoxic potential, and the overall ensemble prediction is determined by majority vote among all trees. All designs were 5 to 7 edits from the training set (Fig. $4 \mathrm{~b}$ ), so we expect around $70 \%$ of the predictions to be accurate (Fig. 3c). The oligonucleotide seth was predicted as having a high hepatotoxic potential (seth in Fig. 4a and 4c). For experimental verification, we chose the redesigned sequence with the lowest possible prediction score, and therefore most likely to have a low hepatotoxic potential, ( $r 3$ in Fig. $4 a$ and $4 c$ ) as well as two additional designs with intermediate predictions: one predicted as having a high hepatotoxic potential ( $r 1$ in Fig. $4 \mathrm{a}$ and $4 \mathrm{c}$ ), the other as having a low hepatotoxic potential ( $r 2$ in Fig. 4a and 4c). We synthesized these four oligonucleotides and characterized their hepatotoxic potential in the 16-day

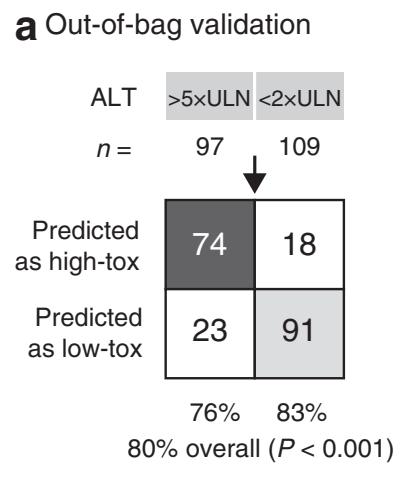

C Stratified 10-fold cross validation as function of distance mouse model. Consistent with earlier reports (Seth et al., 2009) seth induced ALT elevations 6.7 times above saline (Fig. 4c), and the redesigned oligonucleotide, $r 1$, predicted as having a high hepatotoxic potential, indeed induced ALT levels 29 times above saline. Importantly, the redesigned oligonucleotides $r 2$ and $r 3$ predicted as having a low hepatotoxic potential were both confirmed to result in ALT levels below $2 \times$ ULN. These results exemplify that the random forests classifier can successfully guide lead-optimization efforts to identify subtly modified oligonucleotides ( $r 2$ and $r 3$ ), that have much lower hepatotoxic potential than the original oligonucleotide (seth). Furthermore, inspection of these four sequences show, that in particular the addition of an AT in the $5^{\prime}$ gap-region of the $r 2$ and $r 3$ oligonucleotides is responsible for the reduced hepatotoxic potential (compare $r 1$ with $r 2$ ). Inspection of Fig. $2 b$ shows that, indeed, this dinucleotide is associated with reduced hepatotoxic potential. However, the association is not statistically significant when evaluated across all oligonucleotides containing AT (Fig. 2b). Still, the random forests classifier, which also takes interactions between dinucleotides into account, is able to correctly predict that the inclusion of AT in these particular oligonucleotides will reduce the hepatotoxic potential significantly. For additional examples of oligonucleotides where the predicted hepatotoxic potential is confirmed experimentally, see Supplementary Table S6.

\section{Discussion}

Here, we used machine learning to evaluate the extent to which the hepatotoxic potential of oligonucleotides can be predicted from sequence and modification pattern. Using the random forests algorithm for classification, and encoding oligonucleotides as dinucleotide counts (with 4 DNAs and 4 LNAs, 64 dinucleotides are possible), as many as 17 dinucleotides were identified which alone, or in combination, were highly informative with respect to distinguishing between oligonucleotides with low or high potential for hepatotoxicity. A Levenshtein-edit distance measure was furthermore established that allowed stratification of the predictive

FIG. 3. Sequence-based prediction of hepatotoxic potential of oligonucleotides using random forests. (a) Out-of-bag estimate of classification performance. Results are shown in a modified $2 \times 2$ contingency table that were used to calculate the percentage of predictions that agreed with experimentally established classes of high and low hepatotoxic potential (correct predictions are colored dark gray and light gray. Specificity, sensitivity, and overall accuracy calculated). $P$ values were calculated using Fisher's exact test. (b) Top 17 most important dinucleotides for classification, as evaluated by having a mean decrease in Gini index larger than 2. (c) Stratified 10-fold cross validation estimate of classification performance for oligonucleotides that are close, medium, or far away, respectively, as measured by the number of edits (Levensthein distance), from the closest oligonucleotide in the training set. (d) Prediction of hepatotoxic potential in an independent validation set of 13 oligonucleotides inducing ALT $>5 \times$ ULN at lower total doses than $75 \mathrm{mg} / \mathrm{kg}$ (between $25 \mathrm{mg} / \mathrm{kg}$ and $60 \mathrm{mg} / \mathrm{kg}$ ), and 10 oligonucleotides where ALT $<2 \times$ ULN at higher total doses than $75 \mathrm{mg} / \mathrm{kg}$ (between $100 \mathrm{mg} / \mathrm{kg}$ and $125 \mathrm{mg} / \mathrm{kg}$ ). (e) Distribution of Levenshtein distances for each of the 23 oligonucleotides in the validation set to the closest oligonucleotides in the training set. 


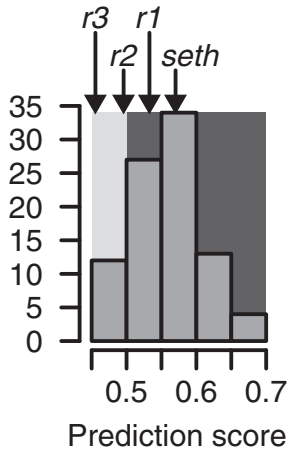

b
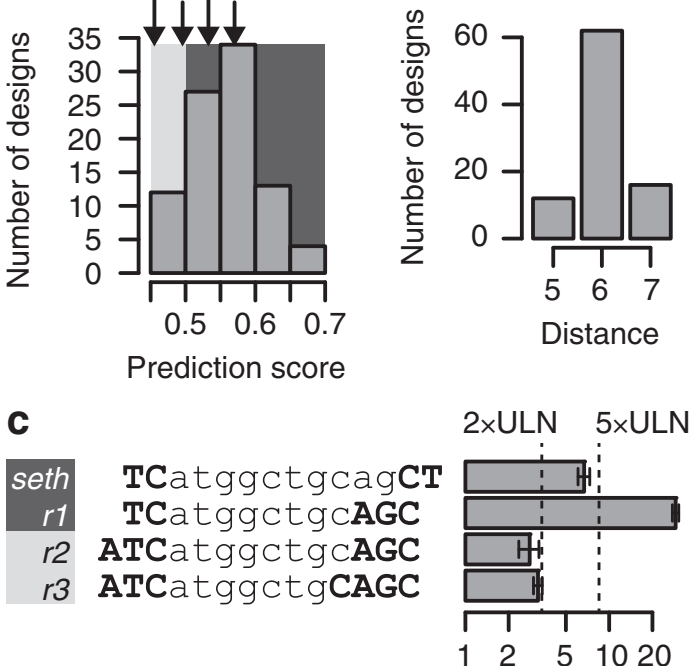

ALT (treatment/saline)

FIG. 4. Model prediction and experimental evaluation of redesigned oligonucleotides. (a) Distribution of hepatotoxicity prediction scores for redesigned oligonucleotides targeting PTEN. Oligonucleotides with scores $<0.5$ are judged as having low hepatotoxic potential (dark gray region), and those with scores $>0.5$ as having high hepatotoxic potential (light gray region). (b) Distribution of Levensthein distances to training set for redesigned oligonucleotides targeting PTEN. (c) Sequence for oligonucleotide seth, and redesigned variants $(r 1, r 2$, and $r 3)$, and ALT levels when dosed $5 \times 15 \mathrm{mg} / \mathrm{kg}$ over 16 days in NMRI mice. LNA shown in upper-case letters, DNA in lower-case letters. Error bars, standard error of the mean.

performance based on the distance between the oligonucleotide to be predicted, and the set of oligonucleotides used to train the classifier. Most oligonucleotides, specifically those less than 6 edits away from the nearest member of the training set, were found to be predicted with at least $80 \%$ accuracy as evaluated by 10 -fold cross validation. When oligonucleotides were further than 6 edits away, the estimated predictive performance was at $69 \%$ as evaluated by 10 -fold cross validation. In an independent set of 23 oligonucleotides, the majority of which were 6 or more edits away, $74 \%$ were predicted correctly. Moreover, when using the trained random forests classifier to guide the efficient redesign of an oligonucleotide demonstrated to have a high hepatotoxic potential, several new designs were identified, and a low hepatotoxic potential confirmed experimentally for two of them. Taken together, these findings indicate that descriptors of sequence composition and modification pattern, here dinucleotide counts, can capture and quantify characteristics of the oligonucleotides associated with hepatic injury, and thereby distinguish with high accuracy and robustness between oligonucleotides with low and high hepatotoxic potential. Including such a predictor in the drug discovery process is expected to reduce the attrition rate due to observed hepatotoxicity in rodents.

The results presented here do not directly elucidate the mechanisms of hepatotoxicity. We speculate, however, that the hepatotoxic potential is related to the propensity of an oligonucleotide to bind to certain proteins. That oligonucleotides can interact with specific proteins is well known (Bennet and Swayze, 2010), and these interactions can be sequence dependent, as is the case with Toll-like receptors binding oligonucleotides containing cg dinucleotides (Krieg, 2006). Another, endogenous, example is found with the genetic disorder myotonic dystrophy, a dominantly inherited disease that is the most common cause of muscular dystrophy in adults. Here, microsatellite expansions result in formation of a double-stranded hairpin structure of repeated cug trinucleotides in the 3'UTR of the dystrophia myotonica-protein kinase gene (DMPK). The protein muscleblind-like splicing regulator 1 (MBNL1) is sequestered to this hairpin structure depleting it from the nucleoplasm. The downstream effects include disruption of alternative splicing, mRNA translation and mRNA decay (Lee and Cooper, 2009). Similarly, our observations are in accord with a model where yet unidentified proteins exist, which when interacting in a sequence and modification-specific manner with an oligonucleotide, induce hepatotoxicity in mice. A possible next step is to employ pulldown methods (Rigo et al., 2012) to characterize the proteins binding preferentially to oligonucleotides with either low or high hepatotoxic potential.

Until now, a main differentiator between small molecule drugs and oligonucleotides has been that oligonucleotides can be designed efficiently to ensure a high proportion of hits when it comes to binding and inhibiting their intended molecular target. With this work we show that the sequencebased nature of oligonucleotides allows a simple decomposition, which, given sufficient amounts of data, enables prediction also of complex, nonhybridization-mediated properties like hepatotoxicity. We expect that this approach is generalizable to other complex properties such as bioavailability and biodistribution, as well as other types of toxicity.

\section{Acknowledgments}

For their excellent technical assistance, we would like to thank Camilla T. Haugaard, Lisbeth Bang, and Rikke Sølberg, for handling of animal facilities, Bo R. Pedersen, Marianne B. Mogensen, for synthesis and formulation of LNA-modified oligonucleotides, Bettina Nordbo, Lene S Jørgensen, Charlotte Øverup, and Jette D Hedegaard, for liver and serum measurements; Stine Møllerud for data collection; and Roger Burnett for histopathology evaluations. Additionally, we are grateful to Bo Hansen, Yann Tessier and Mark Turner for valuable discussions and support in the preparation of the manuscript.

\section{Author Disclosure Statement}

P.H. Hagedorn, S. Ottosen, S. Kammler, N.F. Nielsen, A.M. Høg, M. Hedtjärn, M. Meldgaard, M.R. Møller, H. Ørum, T. Koch, and M. Lindow are employees of Santaris Pharma, a company that is developing LNA-modified oligonucleotides for therapeutic purposes. The work is partially funded by a grant from the Danish Strategic Research Council.

\section{References}

BENNETT, C.F., and SWAYZE, E.E. (2010). RNA targeting therapeutics: molecular mechanisms of antisense oligonucle- 
otides as a therapeutic platform. Annu. Rev. Pharmacol. Toxicol. 50, 259-293.

BENIGNI, R., and GIULIANI, A. (2003). Putting the predictive toxicology challenge into perspective: reflections on the results. Bioinformatics 19, 1194-1200.

BREIMAN, L. (2001). Random forests. Mach. Learn. 45, 5-32.

BUREL, S., and HENRY, S. (2010). Compounds and methods for modulating toxic and proinflammatory effects. Patent. International publication number WO2010108035A1.

DUDA, R.O., HART, P.E., and STORK, D.G. (2001). Pattern Classification, 2nd ed., (John Wiley, New York), pp. 394-443.

DURBIN, R., EDDY, S. R., KROGH, A., and MITCHISON, G. (1998). Biological Sequence Analysis: Probabilistic Models of Proteins and Nucleic Acids. (Cambridge University Press, Cambridge, UK), pp. 46-79.

ELMÉN, J., LINDOW, M., SILAHTAROGLU, A., BAK, M., CHRISTENSEN, M., LIND-THOMSEN, A., HEDTJÄRN, M., HANSEN, J.B., HANSEN, H.F., STRAARUP, E.M., et al. (2008). Antagonism of microRNA-122 in mice by systemically administered LNA-antimiR leads to up-regulation of a large set of predicted target mRNAs in the liver. Nucleic Acids Res. 36, 1153-1162.

GUPTA, N., FISKER, N., ASSELIN, M.-C., LINDHOLM, M.W., ROSENBOHM, C., ØRUM, H., ELMÉN, J., SEIDAH, N.G., and STRAARUP, E.M. (2010). A locked nucleic acid antisense oligonucleotide (LNA) silences PCSK9 and enhances LDLR expression in vitro and in vivo. PLoS One 5, e10682.

HENRY, S., STECKER, K., BROOKS, D., MONTEITH, D., CONKLIN, B. and BENNETT, C.F. (2000). Chemically modified oligonucleotides exhibit decreased immune stimulation in mice. J. Pharmacol. Exp. Ther. 292, 468-479.

HENRY, S.P., KIM, T.-W., KRAMER-STICKLUND, K., ZANARDI, T.A., FEY, R.A., and LEVIN, A.A. (2007). Toxicologic properties of 2'-O-methoxyethyl chimeric antisense inhibitors in animals and man. In: S. T. Crooke, Antisense Drug Technology. (CRC Press, Boca Raton, FL), pp. 327-365.

HERT, J., IRWIN, J.J., LAGGNER, C., KEISER, M.J., and SHOICHET, B.K. (2009). Quantifying biogenic bias in screening libraries. Nature Chem. Biol. 5, 479-483.

HILDEBRANDT-ERIKSEN, E.S., AARUP, V., PERSSON, R., HANSEN, H.F., MUNK, M.E. and ØRUM, H. (2012). A locked nucleic acid oligonucleotide targeting microRNA 122 is well tolerated in cynomolgus monkeys. Nucleic Acid Ther. 22, 152-161.

JANSSEN, H.L., REESINK, H.W., LAWITZ, E.J., ZEUZEM, S., RODRIGUEZ-TORRES, M., PATEL K., VAN DER MEER, A. J., PATICK, A. K., CHEN, A., ZHOU, Y., et al. (2013). Treatment of HCV infection by targeting microRNA. N. Engl. J. Med. 368, 1685-1694.

KOSHKIN, A.A., SINGH, S.K., NIELSEN, P., RAJWANSHI, V.K., KUMAR, R., MELDGAARD, M., OLSEN, C.E. and WENGEL, J. (1998). LNA (locked nucleic acids): synthesis of the adenine, cytosine, guanine, 5-methylcytosine, thymine and uracil bicyclonucleoside monomers, oligomerisation, and unprecedented nucleic acid recognition. Tetrahedron 54, 3607-3630.

KRIEG, A.M. (2006). Therapeutic potential of Toll-like receptor 9 activation. Nat. Rev. Drug Discov. 5, 471-484.

LEE, J.E., and COOPER, T.A. (2009). Pathogenic mechanisms of myotonic dystrophy. Biochem. Soc. Trans. 37, 1281-1286.

LEVENSHTEIN, V.I. (1966). Binary codes capable of correcting deletions, insertions, and reversals. Soviet Phys. Dokl. 10, 707-710.
LEVIN, A.A. (1999). A review of the issues in the pharmacokinetics and toxicology of phosphorothioate antisense oligonucleotides. Biochim. Biophys. Acta 1489, 69-84.

LINDHOLM, M.W., ELMÉN, J., FISKER, N., HANSEN, H.F., PERSSON, R., MØLLER, M.R., ROSENBOHM, C., ØRUM, H., STRAARUP, E.M. and KOCH, T. (2012). PCSK9 LNA antisense oligonucleotides induce sustained reduction of LDL cholesterol in nonhuman primates. Mol. Ther. 20, 376-381.

LINDOW, M., VORNLOCHER, H.-P., RILEY, D., KORNBRUST, D.J., BURCHARD, J., WHITELEY, L.O., KAMENS, J., THOMPSON, J.D., NOCHUR, S., YOUNIS, H., et al. (2012). Assessing unintended hybridization-induced biological effects of oligonucleotides. Nat. Biotechnol. 30, 920-923.

LIPINSKI, C.A., LOMBARDO, F., DOMINY, B.W., and FEENEY, P.J. (2001). Experimental and computational approaches to estimate solubility and permeability in drug discovery and development settings. Adv. Drug Deliv. Rev. 46, 3-26.

LIPINSKI, C.A., and HOPKINS, A.L. (2004). Navigating chemical space for biology and medicine. Nature 432, 855-861.

LOW, Y., UEHARA, T., MINOWA, Y., YAMADA, H., OHNO, Y., URUSHIDANI, T., SEDYKH, A., MURATOV, E., KUZ'MIN, V., FOURCHES, D., et al. (2011). Predicting druginduced hepatotoxicity using QSAR and toxicogenomics approaches. Chem. Res. Toxicol. 24, 1251-1262.

OBIKA, S., NANBU, D., HARI, Y., MORIO, K.-I., IN, Y., ISHIDA, T., and IMANISHI, T. (1997). Synthesis of 2'-O,4'-Cmethyleneuridine and -cytidine. Novel bicyclic nucleosides having a fixed C3, -endo sugar puckering. Tetrahedron Lett. 38, 8735-8738.

PETERS, T.S. (2005). Do preclinical testing strategies help predict human hepatotoxic potentials? Toxicol. Pathol. 33, 146-154.

PLATT, J.C. (1999). Probabilistic outputs for support vector machines and comparisons to regularized likelihood methods. In: A. Smola, A.,Bartlett, P., Schölkopf, B., and Shuurmans, D., eds., Advances in Large Margin Classifiers. (MIT Press, Cambridge, MA).

RIGO, F., HUA, Y., CHUN, S.J., PRAKASH, T.P., KRAINER, A.R., and BENNETT, C.F. (2012). Synthetic oligonucleotides recruit ILF2/3 to RNA transcripts to modulate splicing. Nat. Chem. Biol. 8, 555-561.

SCHUBERT, D., LEVIN, A. A., KORNBRUST, D., BERMAN, C. L., CAVAGNARO, J., HENRY, S., SEGUIN, R., FERRARI, N., and SHREWSBURY, S.B. (2012). The Oligonucleotide Safety Working Group (OSWG). Nucleic Acid Ther. 4, 211-212.

SETH, P.P., SIWKOWSKI, A., ALLERSON, C.R., VASQUEZ, G., LEE, S., PRAKASH, T.P., WANCEWICZ, E.V., WITCHELL, D. and SWAYZE, E.E. (2009). Short antisense oligonucleotides with novel $2^{\prime}-4^{\prime}$ conformationaly restricted nucleoside analogues show improved potency without increased toxicity in animals. J. Med. Chem. 52, 10-13.

STANTON, R., SCIABOLA, S., SALATTO, C., WENG, Y., MOSHINSKY, D., LITTLE, J., WALTERS, E., KREEGER, J., DIMATTIA, D., CHEN, T., et al. (2012). Chemical Modification Study of Antisense Gapmers. Nucleic Acid Ther. 22, 344-359.

STEIN, C.A., SUBASINGHE, C., SHINOZUKA, K., and COHEN, J.S. (1988). Physicochemical properties of phosphorothioate oligodeoxynucleotides. Nucleic Acids Res. 16, 3209-3221.

STRAARUP, E.M., FISKER, N., HEDTJÄRN, M., LINDHOLM, M.W., ROSENBOHM, C., AARUP, V., HANSEN, H.F., ØRUM, H., HANSEN, J.B.R., and KOCH, T. (2010). Short locked nucleic acid antisense oligonucleotides potently reduce 
apolipoprotein B mRNA and serum cholesterol in mice and non-human primates. Nucleic Acids Res. 38, 7100-7111.

SWAYZE, E.E., SIWKOWSKI, A.M., WANCEWICZ, E.V., MIGAWA, M.T., WYRZYKIEWICZ, T.K., HUNG, G., MONIA, B.P., and BENNETT, C.F. (2007). Antisense oligonucleotides containing locked nucleic acid improve potency but cause significant hepatotoxicity in animals. Nucleic Acids Res. 35, 687-700.

SWAYZE, E.E., and SIWKOWSKI, A.M. (2009). Oligomeric compounds composing bicyclic nucleosides and having reduced toxicity. Patent. International publication number WO09124295A2.

WANG, R., LAI, L., and WANG, S. (2002). Further development and validation of empirical scoring functions for structure- based binding affinity prediction. J. Comput. Aided Mol. Des. $16,11-26$

\author{
Address correspondence to: \\ Morten Lindow, PhD \\ Informatics Department \\ Santaris Pharma A/S \\ Horsholm DK2970 \\ Denmark
}

E-mail: morten.lindow@santaris.com

Received for publication June 6, 2013; accepted July 1, 2013. 